\title{
07.2;07.3
}

\section{Гетероструктуры $\mathrm{Ga}_{x} \ln _{1-x} \mathrm{As}_{y} \mathrm{Bi}_{z} \mathrm{Sb}_{1-y-z} / \mathrm{lnSb}$ для фотоприемных устройств $(\lambda=6-12 \mu \mathrm{m})$}

\author{
() Л.С. Лунин ${ }^{1}$, М.Л. Лунина ${ }^{1}$, А.С. Пащенко ${ }^{1,2}$, Д.Л. Алфимова ${ }^{1}$, O.С. Пащенко $^{1}$ \\ ${ }_{1}^{1}$ Федеральный исследовательский центр Южный научный центр РАН, Ростов-на-Дону, Россия \\ ${ }^{2}$ Южно-Российский государственный политехнический университет (НПИ) им. М.И. Платова, г. Новочеркасск, Россия \\ E-mail: lunin_Is@mail.ru
}

Поступило в Редакцию 30 апреля 2019г.

В окончательной редакции 8 мая 2019г.

Принято к публикации 13 мая 2019 г.

\begin{abstract}
Методом зонной перекристаллизации градиентом температуры получены изопараметрические гетероструктуры $\mathrm{Ga}_{x} \mathrm{In}_{1-x} \mathrm{As}_{y} \mathrm{Bi}_{z} \mathrm{Sb}_{1-y-z} / \mathrm{InSb}$ для фотоприемных устройств, работающих в диапазоне длин волн 6-12 $\mu \mathrm{m}$. Введение висмута в твердый раствор GaInAsSb позволяет осуществить уменьшение ширины запрещенной зоны $E_{g}$ и соответственно расширить спектральный диапазон до $12 \mu \mathrm{m}$, а также сдвинуть максимум фоточувствительности в длинноволновую область.
\end{abstract}

Ключевые слова: изопараметрические гетероструктуры, фотоприемники, ИК-детекторы, абсолютная спектральная чувствительность, вольт-ваттная характеристика.

DOI: 10.21883/PJTF.2019.16.48152.17863

Прогресс инфракрасной оптоэлектроники обусловливает необходимость поиска технологических режимов получения гетероструктур со спектрами пропускания в области менее $0.15 \mathrm{eV}$. Такие структуры перспективны в качестве элементной базы диодов, работающих в дальней ИК-области $(\lambda>8 \mu \mathrm{m})$. Приборы на их основе могут успешно использоваться в волоконной оптике, технике регистрации слабых излучений, тепловизорах и т.д.

Уменьшение ширины запрещенной зоны $E_{g}$ и соответствующий сдвиг максимума фоточувствительности в длинноволновую область можно осуществлять с использованием в гетероструктурах изовалентных компонентов (таких как висмут), активно влияющих на зонную структуру твердых растворов, что открывает широкие возможности в управлении спектром люминесценции $[1,2]$.

Возможность получения положительного эффекта с Вi была подтверждена экспериментально на примере выращенных эпитаксиальных слоев InAsBiSb на InSb [3-6]. Однако в данном случае в качестве лимитирующих технологических факторов выступают ограничение растворимости висмута в растворах-расплавах $\mathrm{A}^{3} \mathrm{~B}^{5}$ и рост числа структурных дефектов, обусловленных различием подложки и слоя по параметрам решетки и коэффициенту термического расширения (КТР). Достаточно эффективным решением этой проблемы является введение в твердый раствор InAsBiSb пятого компонента - галлия.

Цель настоящей работы состоит в получении и исследовании твердых растворов GaInAsBiSb, изопараметрических антимониду индия, для фотоприемных устройств $(\lambda=6-12 \mu \mathrm{m})$.

Гетероструктуры GaInAsBiSb/InSb были получены методом зонной перекристаллизации градиентом температуры (ЗПГТ). Метод ЗПГТ характеризуется предельно малыми пересыщениями на фронте кристаллизации, высокой изотермичностью процесса и низкими значениями концентрационного переохлаждения [7]. Отличительной особенностью ЗПГТ является наличие твердого источника, с помощью которого происходит непрерывная подпитка жидкой фазы в процессе роста эпитаксиального слоя. Поэтому метод ЗПГТ позволяет выращивать высокосовершенные слои простых и сложных полупроводников с заданным распределением компонентов и примесей и не использовать при этом толстые слои жидкой фазы. Кроме того, при ЗПГТ легирование и кристаллизационная очистка происходят одновременно. Кристалл очищается вдоль траектории зоны от примесей, которых в жидкой фазе первоначально достаточно мало, и легируется атомами элементов, входящих в состав зоны, если достаточно мала их концентрация в твердой фазе. В определенных случаях конечный результат легирования и очистки методом ЗПГТ проявляется в виде комбинированного эффекта, в котором оба процесса непрерывно связаны. Поэтому очень важно контролировать распределение примесей в эпитаксиальных слоях, которое оказывает существенное влияние на электрофизические параметры гетероструктуры. Взаимодействие их электронных спектров с энергетической структурой многокомпонентного эпитаксиального слоя (GaInAsBiSb) и определяет эксплуатационные характеристики материалов.

Для определения типа проводимости, концентрации основных носителей $(n)$ и их подвижности $(\mu)$ использовалась стандартная методика холловских измерений.

В таблице приведены электрические характеристики твердого раствора $\mathrm{Ga}_{x} \mathrm{In}_{1-x} \mathrm{As}_{y} \mathrm{Bi}_{z} \mathrm{Sb}_{1-y-z} / \mathrm{InSb}$. Из таблицы следует, что электрические характеристики твердого раствора существенно зависят от содержания висмута, что, по-видимому, объясняется различными механизмами вхождения его атомов в кристаллическую решетку. Как показали эксперименты, при $T=300 \mathrm{~K}$ по- 
Значения концентрации $(n)$ и подвижности $(\mu)$ носителей в гетероструктуре $\mathrm{Ga}_{x} \operatorname{In}_{1-x} \mathrm{As}_{y} \mathrm{Bi}_{z} \mathrm{Sb}_{1-y-z} / \mathrm{InSb}_{\text {при }}$ различном содержании висмута в твердой фазе $X_{\mathrm{Bi}}^{S}$

\begin{tabular}{c|c|c|c|c}
\hline \multirow{2}{*}{$X_{\mathrm{Bi}}^{S}$, mol. frac. } & \multicolumn{2}{|c|}{$T=77 \mathrm{~K}$} & \multicolumn{2}{|c}{$T=300 \mathrm{~K}$} \\
\cline { 2 - 5 } & $n \cdot 10^{-16}, \mathrm{~cm}^{-3}$ & $\mu \cdot 10^{-4}, \mathrm{~cm}^{2} /(\mathrm{V} \cdot \mathrm{s})$ & $n \cdot 10^{-16}, \mathrm{~cm}^{-3}$ & $\mu \cdot 10^{-4}, \mathrm{~cm}^{2} /(\mathrm{V} \cdot \mathrm{s})$ \\
\hline 0.005 & 1.2 & 89 & 1.4 & 2.8 \\
0.008 & 1.2 & 106 & 1.5 & 3.0 \\
0.010 & 1.5 & 136 & 2.0 & 3.3 \\
0.012 & 1.8 & 172 & 2.3 & 3.5 \\
0.014 & 1.9 & 180 & 2.5 & 3.7 \\
0.016 & 2.1 & 188 & 2.5 & 3.8 \\
0.018 & 2.2 & 198 & 2.7 & 3.9
\end{tabular}

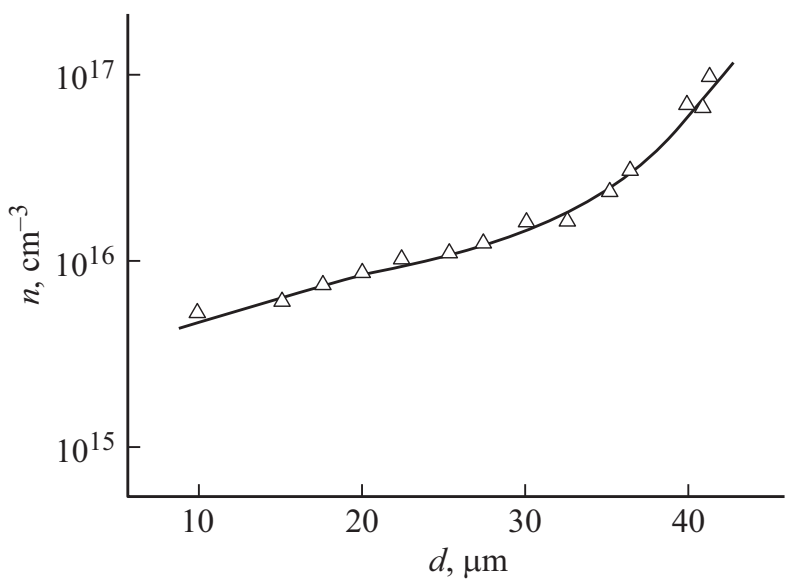

Рис. 1. Распределение концентрации электронов по толщине эпитаксиального слоя $\mathrm{Ga}_{x} \operatorname{In}_{1-x} \mathrm{As}_{y} \mathrm{Bi}_{z} \mathrm{Sb}_{1-y-z} / \mathrm{InSb}$.

движность электронов незначительно зависит от концентрации примеси. Распределение концентрации электронов по толщине гетерослоя показано на рис. 1 . В области $d<30 \mu \mathrm{m}$ наблюдается слабый рост концентрации, в интервале $30<d<35 \mu \mathrm{m}$ имеет место изгиб кривой. Это обусловлено накоплением атомов Вi в междоузлиях приповерхностных участков слоя.

Для максимальной спектральной чувствительности и быстродействия фотоприемников важно обеспечить в первую очередь высокое качество гетероструктуры $\mathrm{Ga}_{x} \mathrm{In}_{1-x} \mathrm{As}_{y} \mathrm{Bi}_{z} \mathrm{Sb}_{1-y-z} / \mathrm{InSb}$.

Поэтому актуальной является задача исследования распределения упругих напряжений в эпитаксиальных слоях GaInAsBiSb(InSb). Исследования проводились на основе стандартного поляризационно-оптического метода. Использовался инфракрасный микроскоп МИК-11 с электронно-оптическим преобразователем ЭОП-4 в поляризованном свете. Анализ лучей проходящего света показал, что упругие напряжения концентрируются в области $<5 \mu \mathrm{m}$ от гетерограницы и принимают в объеме слоя значения, не превышающие $2 \cdot 10^{6} \mathrm{~Pa}$. Величина напряжений в подложке и слое зависит от состава твердого раствора. Результаты расчетов $\sigma\left(X_{\mathrm{Sb}}^{S}\right)$ в подложке и слое при фиксированном $x=0.05$, а также $\sigma\left(X_{\mathrm{Ga}}^{S}\right)$ при $y=0.03$ представлены на рис. $2\left(X_{\mathrm{Sb}}^{S}\right.$ и $X_{\mathrm{Ga}}^{S}-$ мольные доли сурьмы и галлия в твердой фазе соответственно). Значение $z=0.02$ во всех случаях отвечало пределу растворимости висмута в растворе. Основным компонентом, влияющим на величину упругих напряжений, является галлий. В области составов $X_{\mathrm{Ga}}^{S}>0.05$ величина напряжений не превышает $1 \cdot 10^{6} \mathrm{~Pa}$ по всей толщине слоя. Следовательно, введение в твердый раствор InAsBiSb(InSb) пятого компонента (галлия) улучшает согласование параметров решеток и КТР слоя и подложки и уменьшает величину упругих напряжений (рис. 2, $b$ ).

Спектры фоточувствительности $\mathrm{GaInAsBiSb}(\mathrm{InSb})$ при температуре $300 \mathrm{~K}$ представлены на рис. 3, a. Для определения чувствительности гетероструктуры изме-
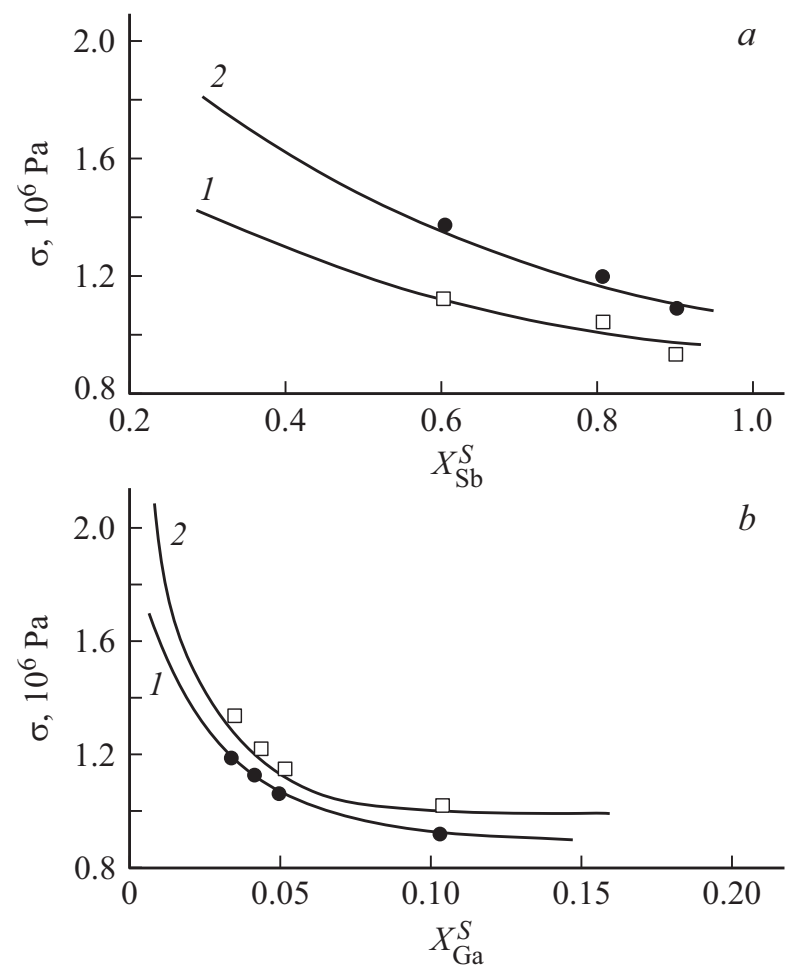

Рис. 2. Зависимость величины упругих напряжений от состава твердой фазы сурьмы $(a)$ и галлия $(b) .1$ - подложка, 2 - эпитаксиальный слой. 


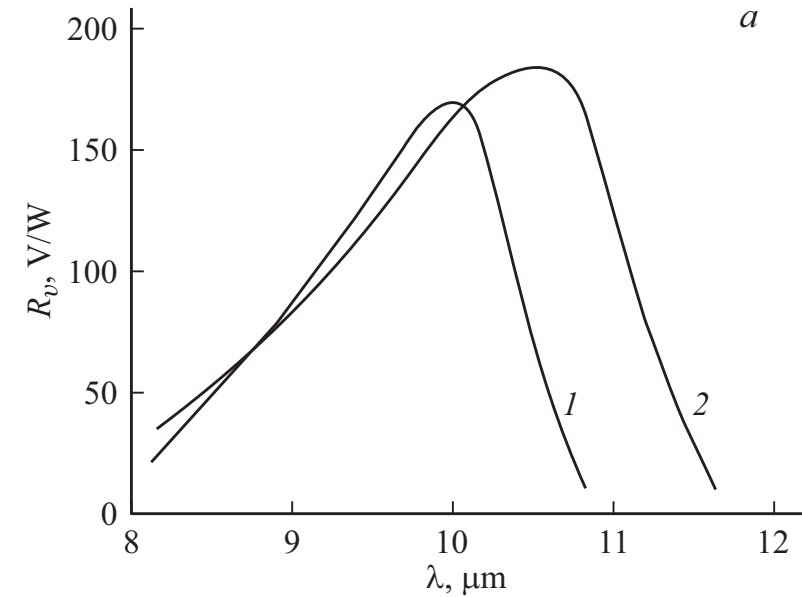

$b$

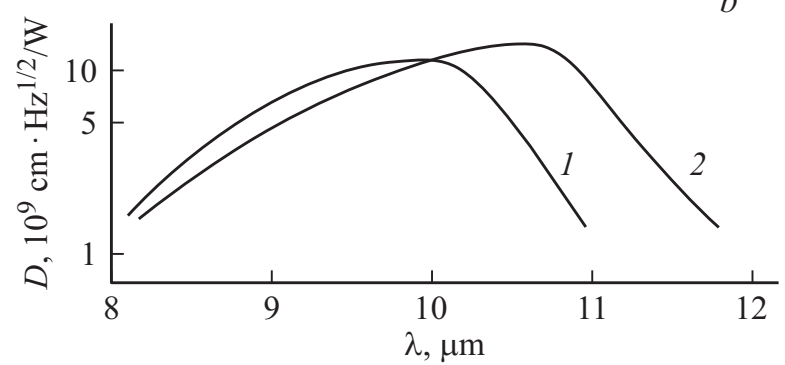

Pис. 3. Спектральные характеристики гетероструктуры GaInAsBiSb/InSb. $a$ - зависимость фоточувствительности $R_{v}(\lambda), b-$ зависимость обнаружительной способности $D(\lambda)$. $1-X_{\mathrm{Bi}}^{S}>0.012,2-X_{\mathrm{Bi}}^{S}>0.02$.

рялась вольт-ваттная чувствительность с использованием модели черного тела по методике [8]. На основе спектральных зависимостей фотопроводимости и спектрального распределения мощности излучения черного тела определялась величина абсолютной спектральной чувствительности фотоприемника. Величина максимальной интегральной чувствительности составила $96 \mathrm{~V} / \mathrm{W}$. Значения монохроматической чувствительности при $\lambda=10 \mu \mathrm{m}$ для $X_{\mathrm{Bi}}^{S}>0.012$ и $\lambda=11 \mu \mathrm{m}$ для $X_{\mathrm{Bi}}^{S}>0.02$ (рис. 3, $a$ ) оказались равными 160 и $180 \mathrm{~V} / \mathrm{W}$ соответственно. Для определения обнаружительной способности фотоприемников были измерены напряжения шумов детектора при различных значениях приложенного напряжения. Измерения проводились на частоте, при которой определялась вольт-ваттная чувствительность $(1 \mathrm{kHz})$. При соотношении сигнал/шум, равном 10 , интегральная обнаружительная способность фотоприемников достигала величины $7.5 \cdot 10^{9} \mathrm{~Hz}^{1 / 2} \cdot \mathrm{W}^{-1}$. Спектральная зависимость детектирующей способности $D$ имеет вид, показанный на рис. $3, b$. Кривые этой зависимости сходны по виду с кривыми вольт-ваттной чувствительности и имеют максимумы при тех же длинах волн, что и спектральная чувствительность. Следует отметить, что с увеличением концентрации висмута в гетероструктурах GaInAsBiSb/InSb расширяется до 12 $\mu \mathrm{m}$ спектральный диапазон как чувствительности $R_{v}(\lambda)$, так и обнаружительной способности $D(\lambda)$ (рис. $3, a, b)$.
Временна́я зависимость фотоответа была исследована при воздействии на гетероструктуру импульсов излучения $\mathrm{CO}_{2}$-лазера. Выходной сигнал регистрировался запоминающим осциллографом. Параметры исходного светового импульса измерялись с помощью фотоэлектронного умножителя с постоянной времени $\tau_{0}=10^{-11} \mathrm{~s}$. Время фотоответа не превышало $2 \mathrm{~ns}$. Динамическое сопротивление гетероперехода находилось в интервале от 600 до $1200 \Omega$.

Таким образом, впервые получены пятикомпонентные висмутсодержащие гетероструктуры GaInAsBiSb/InSb для фотоприемных устройств $(\lambda=6-12 \mu \mathrm{m})$. Проанализированы возможности снижения дефектности гетерограницы и упругих напряжений при изменении состава твердого раствора GaInAsBiSb.

Исследованы спектральные зависимости чувствительности и обнаружительной способности от содержания висмута в интервале длин волн 6-12 $\mu \mathrm{m}$. Показана перспективность их использования для фотоприемных устройств с $\lambda>8 \mu \mathrm{m}$.

\section{Финансирование работы}

Работа выполнена в рамках государственных заданий Федерального исследовательского центра Южного научного центра РАН (номера госрегистрации 01201354240 и АААА-А19-119040390081-2), а также при финансовой поддержке Российского фонда фундаментальных исследований в рамках научного проекта № 17-08-01206 А.

\section{Конфликт интересов}

Авторы заявляют, что у них нет конфликта интересов.

\section{Список литературы}

[1] Riordan N.A., Gogineni C., Johnson S.R., Lu X., Tiedje T., Ding D., Zhang Y.-H., Fritz R., Kolata K., Chatterjee S., Volz K., Koch S.W. // J. Mater. Sci.: Mater. Electron. 2012. V. 23. N 10. P. 1799-1804.

https://doi.org/10.1007/s10854-012-0665-1

[2] Wang L., Zhang L., Yue L., Liang D., Chen X., Li Y., Lu P., Shao J., Wang S. // Crystals. 2017. V. 7. N 3. P. 63. https://doi.org/10.3390/cryst7030063

[3] Алфимова Д.Л., Лунина М.Л., Лунин Л.С., Пащенко А.С., Казакова А.Е. // ФТТ. 2018. Т. 60. В. 7. С. 1277-1282. DOI: 10.21883/FTT.2018.07.46109.194

[4] Song Y., Gu Y., Shao J., Wang S. // Springer Ser. Mater. Sci. 2013. V. 186. P. 1-27. https://doi.org/10.1007/978-1-4614-8121-8_1

[5] Dhar S. // Springer Ser. Mater. Sci. 2013. V. 186. P. 141-165. https://doi.org/10.1007/978-1-4614-8121-8_6

[6] Webster P.T., Shalindar A.J., Schaefer S.T., Johnson S.R. // Appl. Phys. Lett. 2017. V. 111. N 8. P. 082104. https://doi.org/10.1063/1.4994847

[7] Лозовский В.Н., Лунин Л.С., Попов В.П. Зонная перекристаллизация градиентом температуры полупроводниковых материалов. М.: Металлургия, 1987. $232 \mathrm{c.}$

[8] Padalko A.G., Lazarev V.B., Perry F.S. Medium impedance room temperature indium antimonide thin layer detectors // Conf. Lasers and Electro-Optics Europe. IEEE, 1994. P. 336. https://doi.org/10.1109/CLEOE.1994.636588 